\title{
P103 Improved Metabolic Vasoreactivity in the Brain of HM3 Patients and its Underlying Microcirculatory Mechanisms
}

\author{
Eric J. Stöhr ${ }^{1,}$, Ruiping Ji², Koichi Akiyama ${ }^{2}$, Francesco Castagna ${ }^{2}$, Pinsino Alberto ${ }^{2}$, John Cockcroft ${ }^{1}$, \\ Melana Yuzefpolskaya ${ }^{2}$, Reshad Garan ${ }^{2}$, Veli Topkara ${ }^{2}$, Hiroo Takayama ${ }^{2}$, Koji Takeda ${ }^{2}$, Yoshifumi Naka², \\ Paolo Colombo'2, Joshua Willey ${ }^{2}$, Barry J. McDonnell ${ }^{1}$
}

${ }^{1}$ Cardiff Metropolitan University, Cardiff, UK

${ }^{2}$ Columbia University Irving Medical Center, New York City, USA

\section{ABSTRACT}

Background: The MOMENTUM3 trial1 has revealed superiority of the novel HeartMate3 (HM3) left ventricular assist device (LVAD) compared with the HM2, with a significantly reduced occurrence of cerebrovascular accidents. Thus, cerebral autoregulation may be improved in HM3 compared with HM II patients, possibly because of altered microcirculatory haemodynamics associated with the in-built speed modulation ('pulsatility') of the HM3 device.

Methods: Angle-corrected Doppler ultrasound images of the middle cerebral artery (MCA) were recorded before and at the end of a 30s breathhold test in healthy controls $(n=17)$, heart failure (HF $n=18)$, HM2 $(n=10)$ and HM3 $(n=17)$ patients. Microcirculatory haemodynamics as represented by the central retinal artery (CRA) were also quantified (Controls $=33$, $\mathrm{HF}=27, \mathrm{HM} 2=23, \mathrm{HM} 3=31$ ). Data were analysed for Time-Averaged Maximum flow velocity (TAMAX), peak flow velocity $\left(\mathrm{V}_{\max }\right)$, minimum flow velocity $\left(\mathrm{V}_{\min }\right)$, Pulsatility Index (PI) and Resistance Index (RI, Table 1).

Results: Breathhold significantly increased TAMAX, Vmax and Vmin in all groups except HM II patients (Figure 1A). Conversely, PI decreased slightly in all groups while RI was maintained. The greater breathhold response in HM3 compared with HM2 patients was not attributable to the in-built pump-speed modulation (Figure 1B), however, HM3 had a consistently lower RI in the MCA and CRA.

Conclusion: Although reduced compared with healthy controls, HF and HM3 patients have a significantly greater metabolic cerebral vasoreactivity compared with HM2 patients. The $60 \%$ greater diastolic flow velocity in the microcirculation of both LVAD groups compared to healthy controls may alter gas exchange in the microcirculation. Future studies should examine the role of altered RI in HM3 patients.

Table 1 Haemodynamics in the middle cererbral artery (MCA) and the central retinal artery (CRA) between HeartMate2 and HeartMate3 patients

\begin{tabular}{|c|c|c|c|c|c|c|}
\hline & $\begin{array}{l}\text { Healthy } \\
\text { controls } \\
(n=33)\end{array}$ & $\begin{array}{l}\text { Heart failure } \\
\qquad(n=27)\end{array}$ & $\begin{array}{l}\text { HeartMate II } \\
\qquad(n=23)\end{array}$ & $\begin{array}{c}\text { HeartMate3 } \\
\text { (average) } \\
(n=31)\end{array}$ & $\begin{array}{l}\text { HeartMate3 } \\
\text { (no speed } \\
\text { modulation) } \\
\quad(n=31)\end{array}$ & $\begin{array}{c}\text { HeartMate3 } \\
\text { (with speed } \\
\text { modulation) } \\
\quad(n=31)\end{array}$ \\
\hline \multicolumn{7}{|c|}{ Middle cerebral artery } \\
\hline TAMAX $(\mathrm{cm} / \mathrm{s})$ & $58 \pm 15$ & $48 \pm 13$ & $45 \pm 15$ & $48 \pm 19$ & $48 \pm 19$ & $46 \pm 18$ \\
\hline $\mathrm{V}_{\max }(\mathrm{cm} / \mathrm{s})$ & $92 \pm 22$ & $78 \pm 20$ & $55 \pm 19^{* \#}$ & $55 \pm 21^{\star \#}$ & $54 \pm 21^{\star \#}$ & $55 \pm 22^{\star \#}$ \\
\hline $\mathrm{V}_{\min }(\mathrm{cm} / \mathrm{s})$ & $39 \pm 12$ & $29 \pm 11$ & $37 \pm 16$ & $39 \pm 14$ & $44 \pm 16^{\#}$ & $32 \pm 13$ \\
\hline Pulsatility index & $0.88 \pm 0.18$ & $1.05 \pm 0.31^{*}$ & $0.40 \pm 0.24^{* \#}$ & $0.34 \pm 0.14^{* \#}$ & $0.21 \pm 0.12^{* \# \$}$ & $0.56 \pm 0.24^{* \#}$ \\
\hline Resistance index & $0.57 \pm 0.07$ & $0.60 \pm 0.11$ & $0.29 \pm 0.14^{* \#}$ & $0.16 \pm 0.09^{* \# \$}$ & $0.17 \pm 0.10^{* \# \$}$ & $0.14 \pm 0.09^{* \# \$}$ \\
\hline \multicolumn{7}{|c|}{ Central retinal artery } \\
\hline TAMAX (cm/s) & $6 \pm 1$ & $5 \pm 2$ & $6 \pm 3$ & $7 \pm 3$ & $7 \pm 4$ & $6 \pm 4$ \\
\hline $\mathrm{V}_{\max }(\mathrm{cm} / \mathrm{s})$ & $12 \pm 3$ & $11 \pm 6$ & $8 \pm 4^{*}$ & $8 \pm 4^{*}$ & $8 \pm 4^{*}$ & $8 \pm 4^{*}$ \\
\hline $\mathrm{V}_{\min }(\mathrm{cm} / \mathrm{s})$ & $3 \pm 1$ & $3 \pm 1$ & $5 \pm 2$ & $5 \pm 3$ & $6 \pm 3^{\star \#}$ & $4 \pm 4$ \\
\hline Pulsatility index & $1.60 \pm 0.45$ & $1.42 \pm 0.40$ & $0.58 \pm 0.26^{* \#}$ & $0.49 \pm 0.21^{\#}$ & $0.32 \pm 0.17^{\star \#}$ & $0.79 \pm 0.35^{\star \#}$ \\
\hline Resistance index & $0.75 \pm 0.09$ & $0.70 \pm 0.11$ & $0.38 \pm 0.15^{\star \#}$ & $0.22 \pm 0.11^{\text {\#\$ }}$ & $0.24 \pm 0.12^{* \#}$ & $0.20 \pm 0.13^{\star \# \$}$ \\
\hline
\end{tabular}

" $p<0.05$ compared with healthy controls; $p<0.05$ compared with Heart Failure; ${ }^{\$} p<0.05$ compared with HeartMate II. 

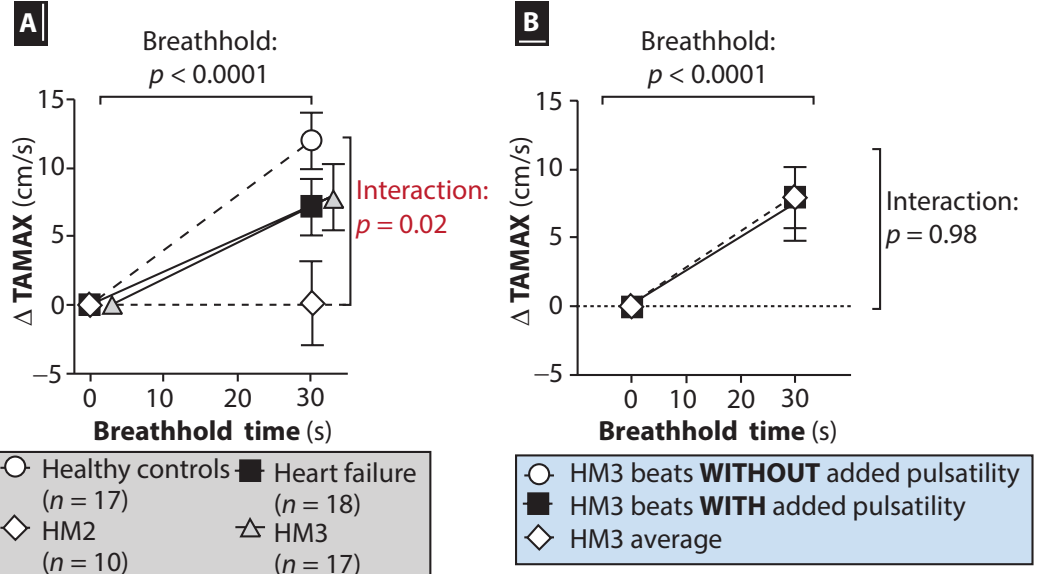

Figure 1 (A) Time-averaged maximum flow velocity in the middle cerebral artery of healthy controls and patient groups in response to a 30-s breathhold test. (B) Breakdown of the responses in HM3 patients, comparing beats with and without added pulsatility.

\section{REFERENCE}

[1] Mehra MR, Uriel N, Naka Y, Cleveland JC, Yuzefpolskaya M, Salerno CT, et al. A fully magnetically levitated left ventricular assist device - final report. N Engl J Med 2019;380:1618-27.

(C) 2019 Association for Research into Arterial Structure and Physiology. Publishing services by Atlantis Press International B.V. This is an open access article distributed under the CC BY-NC 4.0 license (http://creativecommons.org/licenses/by-nc/4.0/). 